Agosto 2012 | www.ocula.it - DOI: 10.12977/ocula12

\title{
I luoghi di Marghera Appunti per una geologia urbana
}

\author{
di Giorgio Coratelli \\ Università di Siena-GiorgioCoratelli@libero.it
}

\begin{abstract}
The places of Marghera. Field notes for urban geology

In this article I analyze the founding of the city of Marghera and the industrial zone of Porto Marghera. I practice an "urban geology" to research the stratification of power relations, constitutive of their realization and dynamics. The first part is on the founding of Marghera: considering Foucault's studies on the governance practices of the space and Deleuze's book of Foucault, I study the project of founding of Marghera, as "garden city" and worikng-class town, to demonstrate the failure of this and describe how the power relations determine the development of residential areas. In the second part I consider Hirschman's studies on developing economies to study the dynamics of power relations in the industial zone, especially in the ages of workers' struggles.
\end{abstract}

\section{Keywords}

Power relations, Disciplinar diagram, Biopolitical diagram, Economic connection, Political connection.

\section{Sommario}

1. Introduzione

2. Porto Marghera e Marghera

3. Movimenti di dissociazione

4. Il "problema di Venezia"

5 . Il governo della città

6. Per una geologia di Marghera

7. Per una geologia di Porto Marghera

Bibliografia 


\section{Ocula ${ }^{13}$}

Architettura e politica: un incrocio di sguardi | Giorgio Coratelli, I luoghi di Marghera Appunti per una geologia urbana

\section{Introduzione}

Una configurazione urbanistica non è solo il risultato di un sistema di potere (Crivelli 2008: 77) o di una pianificazione. Un "oggetto urbano" edificio, quartiere, città - può essere pensato come una complessa stratificazione e sedimentazione di rapporti di forze, campo di analisi per una "geologia urbana": una prospettiva storica e culturale che operi sui luoghi non in quanto "luoghi della memoria", ma localizzando le tracce che conducono alla loro costituzione. Assumiamo il termine "geologia"1 per designare l'operazione di scomposizione e ricomposizione di un luogo, alla ricerca delle dinamiche e delle forme di attualizzazione dei rapporti di forze.

Oggetto di questo tentativo di "geologia urbana" è una parte della terraferma veneziana: la zona portuale e industriale di Porto Marghera e la zona residenziale di Marghera. L'atto di fondazione di Porto Marghera e della congiunta area residenziale si inscrive nel progetto di risoluzione del cosiddetto "problema di Venezia" - non un problema unico, come vedremo più avanti, ma un insieme di questioni - portuale, industriale ed edilizia connesse fra loro.

Nella prima parte proporrò una periodizzazione in quattro fasi degli eventi storici a partire dall'ipotesi di un movimento di dissociazione che conduce Marghera verso la ricerca di una nuova identità, fortemente differenziata rispetto alla zona industriale, e Porto Marghera verso il problema della riqualificazione.

Queste coordinate orientano lo sguardo geologico nella lettura delle dinamiche dei rapporti di forze. Il diagramma - nozione importante sia in Foucault che in Deleuze - è la "carta" dei rapporti di forze che lo sguardo geologico cerca di descrivere. Mi concentrerò sulla prima fase di Marghera per mostrare come i luoghi che compongono il quartiere non siano il risultato di un progetto mal eseguito, di un'anarchia urbanistica o il prodotto dell'esercizio di un potere alto, bensì l'attualizzazione per deviazioni e differenziazioni di molteplici rapporti di forze. I rapporti tra forze e resistenze non avvengono solo nel segno di una contro-condotta che si oppone a una condotta, di tattiche che affrontano le strategie nei luoghi dove queste operano; le resistenze sono anche forze differenti per intensità e per le diverse posizioni attanziali che gli attori possono ricoprire.

Nelle conclusioni mi soffermerò sullo statuto dell'oggetto e la proposta analitica per una geologia della zona industriale di Porto Marghera.

\section{Porto Marghera e Marghera}

L'atto di fondazione di Porto Marghera risale al 1917: Porto Marghera nasce come sistemazione sulla terraferma del porto di Venezia e come "zona franca” per l'insediamento di industrie pesanti. L'atto è preceduto da quindici anni di dibattiti intorno al "problema di Venezia". La zona diventa

${ }^{1}$ Per il concetto di "geologia" rimando a Deleuze e Guattari 1980: 83-89 tr. it.

2 | www.ocula.it | agosto 2012 


\section{Ocula ${ }^{13}$}

Architettura e politica: un incrocio di sguardi | Giorgio Coratelli, I luoghi di Marghera

Appunti per una geologia urbana

un importante polo industriale fin dagli anni Trenta, cresce ancora negli anni Sessanta con l'apertura della seconda zona industriale, diventando polo avanzato della chimica. ${ }^{2}$ Dalla seconda metà degli anni Settanta comincia la parabola discendente.

Vicino alla zona industriale sorge il centro abitato di Marghera. Il progetto della zona residenziale, quale quartiere urbano della zona industriale, è del 19223. L'area centrale ha la forma di un triangolo allungato. Osservando la mappa, se ne possono tracciare i lati4: a destra l'ampia via Fratelli Bandiera separa la zona residenziale dalla zona industriale; a sinistra la tangenziale di Mestre separa Marghera dai sobborghi di Catene e di Villabona, la costruzione dei quali fu avviata a partire dagli anni Cinquanta (Nappi 1994: 77), e che a loro volta sono divisi dal centro abitato di Chirignago, frazione di Venezia, dalla linea ferroviaria; a nord via della Libertà e la linea ferroviaria separano Marghera da Mestre; la rotonda a sud, che funge da vertice del triangolo, e la strada statale Romea separano Marghera dal sobborgo di Ca' Sabbioni, di cui parleremo più avanti, e dal centro abitato di Malcontenta che, divisa a metà dal Naviglio del Brenta, è a nord parte della municipalità di Marghera e a sud frazione di Mira.

\section{Movimenti di dissociazione}

Marghera è il quartiere urbano progettato per i lavoratori di Porto Marghera. All'atto di fondazione le due zone sono pensate insieme. Questa relazione sussiste solo sul piano progettuale: nel corso del tempo si assiste a un continuo movimento di dissociazione che ci permette di leggere la storia di Porto Marghera e Marghera in quattro fasi. A queste corrispondono quattro diversi modi di relazione tra le due zone.

Nella prima fase, che chiamerò "prima fase di industrializzazione", e che percorre gli anni Venti e Trenta, zona industriale e portuale e zona residenziale si presentano congiunte. Il nome di "Marghera" indica la nuova zona del porto: Porto Marghera nasce prima di Marghera. Emilio Emmer, tecnico comunale e progettista del centro abitato, parla di «quartiere urbano di Porto Marghera» e «sobborgo giardino di Venezia in terraferma» (Emmer 1922). Il quartiere deve ospitare la popolazione occupata nella nuova area: operai, impiegati, dirigenti, in gran parte, secondo il progetto, provenienti da Venezia.

La seconda è la "grande fase di industrializzazione". Comprende in particolare gli anni Cinquanta e Sessanta, quelli dell'espansione di Porto Marghera e dell'area della chimica. Questa fase è caratterizzata dal tentativo di tenere insieme le due zone: "Marghera" indica l'intero polo industriale; si

2 Per un'introduzione alla storia di Porto Marghera: Chinello 1975 e 1979.

3 Per un'introduzione alla storia di Marghera: Nappi 1994.

4 V. la mappa satellitare all'indirizzo: http://maps.google.it/maps?f $=$ q\&source $=$ s_q\&hl=it\&geocod $=\& q=$ Marghera,+ Venezia\&aq $=0 \& s l l=41.442726,12.392578 \& s s p n=$ $15 \cdot 537829,39.331055 \&$ ie $=$ UTF8\&hq=\&hnear=Marghera, + Venezia,+ Veneto\&ll $=45.46$ $3923,12.224565 \& s p n=0.014207,0.038409 \& \mathrm{t}=\mathrm{h} \& \mathrm{z}=15$.

3 | www.ocula.it | agosto 2012 


\section{Ocula ${ }^{13}$}

Architettura e politica: un incrocio di sguardi | Giorgio Coratelli, I luoghi di Marghera

Appunti per una geologia urbana

costruiscono nuove abitazioni - fra cui il complesso della Montecatini-Cita e la chiesa del Cristo lavoratore (v. Facca 2004: 76-85; Nappi 1994: 78-79). Nello stesso tempo si afferma il distacco tra Marghera e Venezia: la zona industriale è sempre più estranea alla città lagunare, che vive una seconda fase del "problema Venezia": l'esodo della popolazione e l'inesorabile declino (Dorigo 1973).

La "crisi della fase industriale" è il terzo stadio temporale. Comprende il periodo delle lotte operaie e di quartiere, dalla fine degli anni Sessanta fino alla metà degli anni Settanta. Dopo l'apertura della seconda zona industriale, con la costruzione del Nuovo Petrolchimico (fig. 3.1.) e la nascita della Montedison, Porto Marghera è spesso, per sineddoche, indicata come Petrolchimico: è questo il luogo con il maggior numero di occupati, la punta più avanzata degli impianti industriali della zona è della chimica italiana, ma anche uno dei luoghi in cui è più forte l'autonomia operaia. Da questa fase la storia di Porto Marghera diventa soprattutto la storia del Petrolchimico. Lotte operaie e lotte di quartiere segnano una frattura tra le due zone: sebbene si cerchino dei punti in comune, si tratta di lotte che si manifestano con differenti modalità (v. Sacchetto e Sbrogiò 2009; Sbrogiò 1990 in particolare i documenti in appendice).

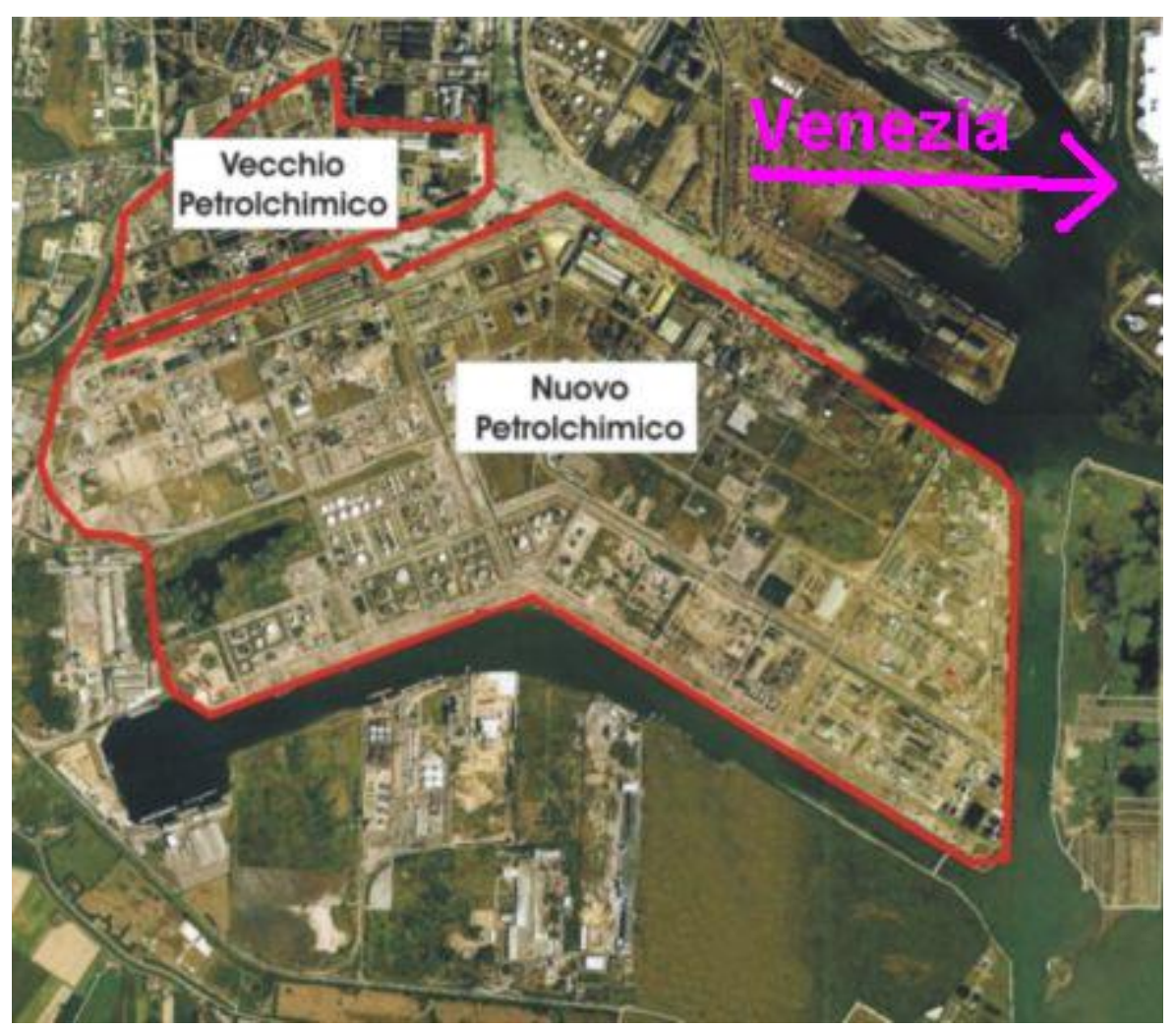

Figura 3.1. Vecchio e Nuovo Petrolchimico (fonte: Confservizi Veneto).

La quarta e ultima fase è la "fase post-industriale" che va dagli anni Ottanta a oggi. L'industria non è più al centro dell'economia territoriale, le 


\section{Ocula $\mathbf{a}^{13}$}

Architettura e politica: un incrocio di sguardi | Giorgio Coratelli, I luoghi di Marghera

Appunti per una geologia urbana

dismissioni si susseguono, cala l'occupazione. L'industria pesante, specie quella della chimica, è percepita come un pericolo per la salute della popolazione. In quest'ultima fase si compie la definitiva dissociazione: emerge il problema ecologico e sanitario, si segnalano i danni causati dalle industrie e i rischi per l'ambiente e per la salute in relazione alle lavorazioni pericolose della chimica e agli scarichi in laguna di sostanze inquinanti (Fabbri 2003). A partire dagli anni Novanta, sono soprattutto quattro gli eventi che segnano questa fase: il processo ai dirigenti Montedison, l'incendio al Petrolchimico del 2002, le manifestazioni cittadine contro le lavorazioni della chimica, il referendum postale del 20065. Chiudono le lavorazioni pericolose, si procede con la bonifica dei siti inquinati, la conversione degli stabilimenti abbandonati per le attività logistiche e interportuali, la ristrutturazione della chimica in un'area più limitata (fig. 3.2. e 3.3.). Marghera muove verso la costruzione di una nuova identità, svincolata dal legame con la zona industriale, in opposizione al progetto della "Grande Venezia", pur rivendicando il nome di città-giardino (Barizza 2009). Sul piano della memoria, ciò non comporta l'oblio di Porto Marghera, ma il superamento del ruolo di sobborgo industriale (Cerasi 2007).

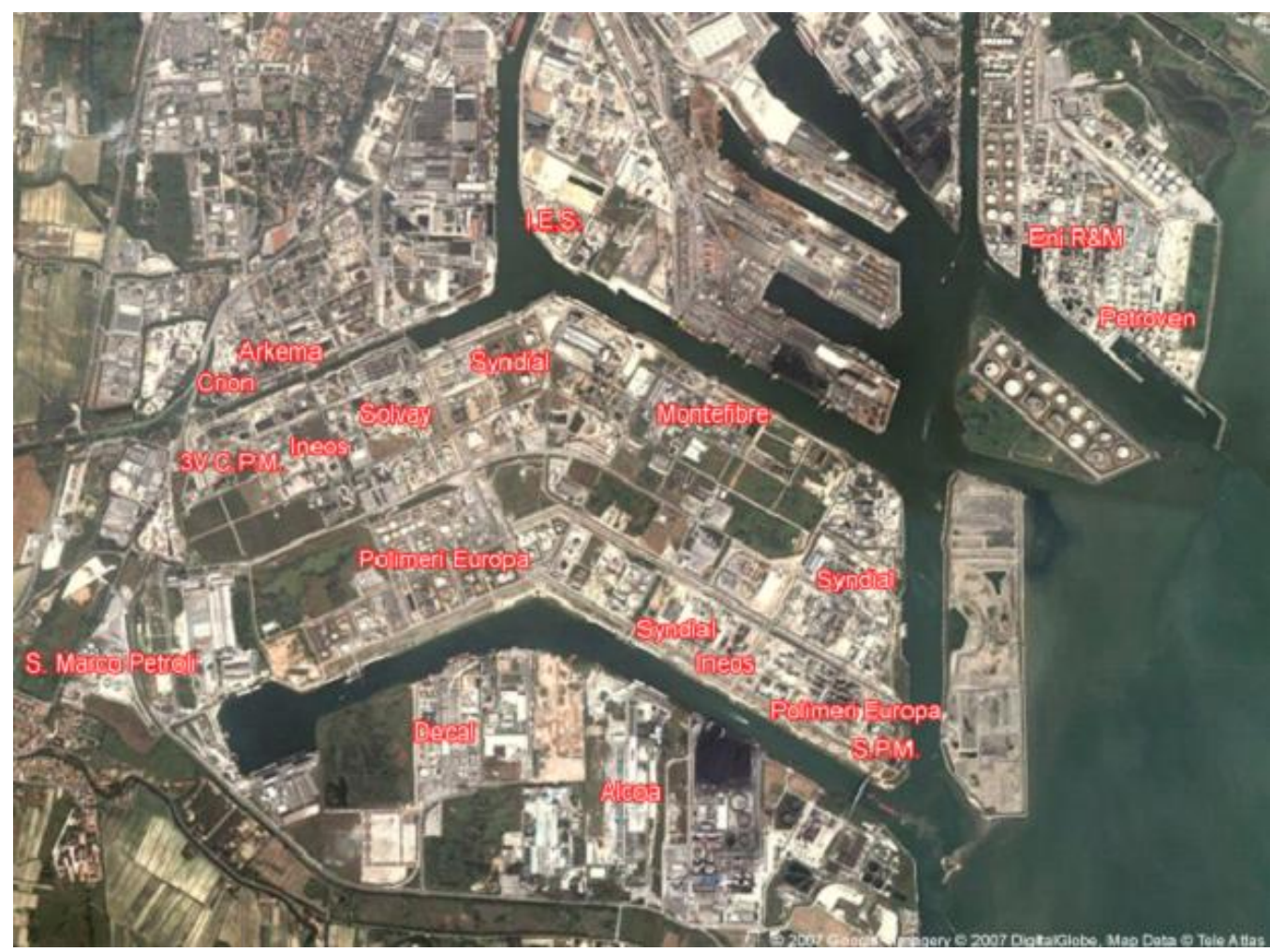

Figura 3.2. Principali aziende di Porto Marghera nel 2007 (fonte: Regione Veneto). Si noti la presenza di numerose aziende nell'area del Nuovo Petrolchimico.

5 Sul processo: Benatelli, Candiello, Favarato 2002 e Casson 2007; sull'incendio del 2002 si vedano le prime pagine di Cerasi 2007; sulle manifestazioni cittadine e il referendum postale: Benatelli, Candiello, Favarato 2006.

5 | www.ocula.it | agosto 2012 


\section{Ocula $\mathbf{a}^{13}$}

Architettura e politica: un incrocio di sguardi | Giorgio Coratelli, I luoghi di Marghera Appunti per una geologia urbana

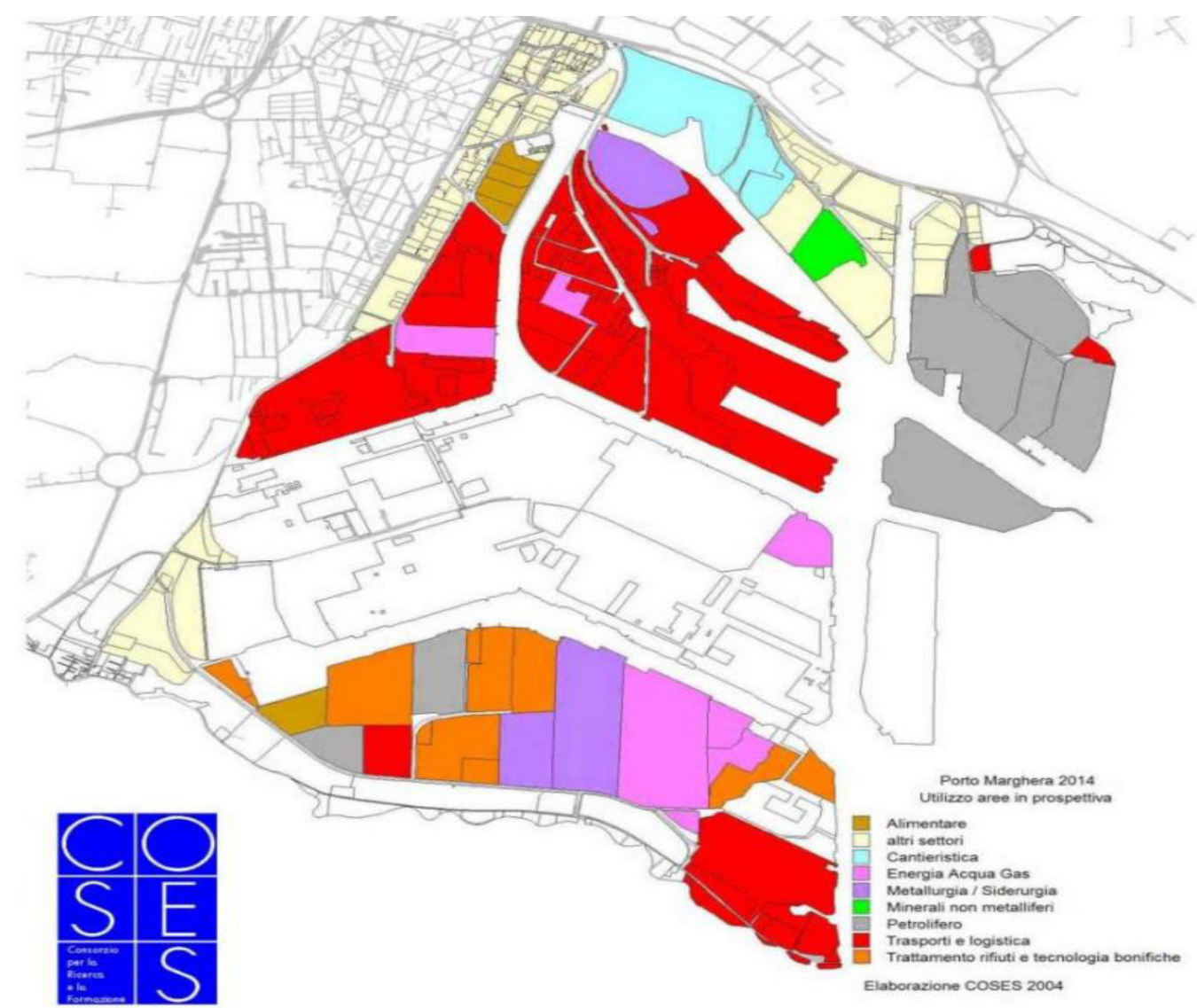

Figura 3.3. Porto Marghera 2014: utilizzo area in prospettiva (fonte: Coses, elaborazione 2004). Si noti il cambio di destinazione dell'area del Nuovo Petrolchimico (parte bianca al centro) e la diffusione delle aree dedicate a trasporto e logistica (zone rosse).

Queste, in sintesi, le quattro fasi: una prima fase di industrializzazione di congiunzione; una grande fase di industrializzazione di non-disgiunzione, dello sviluppo e dell'espansione del polo industriale; una terza fase di noncongiunzione, delle lotte operaie e di quartiere; una quarta fase postindustriale di disgiunzione, delle trasformazioni di Porto Marghera e della ricerca di una nuova identità di Marghera.

Le fasi corrispondono a quattro diversi modi di relazione tra le due zone e di rappresentazione dei luoghi. Si prenda il caso di via Fratelli Bandiera: nella prima fase è concepita come viale alberato per catturare i fumi delle industrie e garantire la convivenza del centro abitato con la zona industriale; nella seconda fase vi si costruiscono nuove abitazioni ed è sede della fondazione della chiesa dedicata a Cristo Lavoratore; nella fase di crisi è 


\section{Ocula ${ }^{13}$}

Architettura e politica: un incrocio di sguardi | Giorgio Coratelli, I luoghi di Marghera

Appunti per una geologia urbana

spesso campo di lotta tra operai e polizia; nella fase post-industriale è cesura netta tra la zona residenziale e la zona industriale ${ }^{6}$.

\section{Il "problema di Venezia"}

Le fasi orientano la lettura delle dinamiche dei rapporti di forze. Mi soffermerò sulla prima fase.

Il dibattito che precede l'atto di fondazione di Porto Marghera vede opporsi due schieramenti (v. Dorigo 1973; Chinello 1979): i sostenitori della sistemazione del porto in terraferma e quelli contrari. Tra i primi, i due principali esponenti sono il capitano Petit e il conte Piero Foscari. Quest'ultimo è l'ideatore del progetto della "Grande Venezia": a Venezia l'arte e la cultura, a Marghera le industrie, a Mestre la vita cittadina. Il progetto è sostenuto dal conte Giuseppe Volpi, artefice della fondazione di Porto Marghera, propugnatore di una "Venezia moderna" erede dei fasti della "Venezia antica", nella cui retorica vi è tutta l'aporia della "Grande Venezia" (Volpi 1939). A tale progetto si oppongono i cosiddetti "neoinsularisti", i quali sostengono che il problema del porto e il problema edilizio possono essere risolti a Venezia. Il motto di questi ultimi è "là dove c'è Venezia, lì deve essere il porto", rovesciato dai primi in "là dove c'è il porto, lì deve essere Venezia".

Il "problema di Venezia" va considerato sotto due aspetti: uno economico, che riguarda il porto e l'industria, l'altro sociale, riguardante le abitazioni. $\mathrm{Al}$ primo posto c'è la questione del porto. La recente costruzione della Stazione Marittima non riesce a contenere gli enormi incrementi dei movimenti portuali. Gran parte delle merce che vi sbarca è merce povera e inquinante carbone e petrolio su tutte. Il porto veneziano, inoltre, è carente dal punto di vista logistico e dei depositi (v. Chinello 1979: 92-93; Baiocco 2002: 41). Al secondo posto c'è la questione dell'industria. A Venezia scarseggiano gli spazi per nuovi insediamenti industriali. C'è poi il problema della presenza di industrie inquinanti nel centro abitato. Nei pressi dell'area dove sorgerà il porto di terraferma è già stata trasferita una fabbrica di concimi (Barizza 2004a: 13); nella stessa area sorgono gli inceneritori (Giovannini 1996: 156). Questi due elementi segnalano il particolare rapporto, di "espulsione", che Venezia intrattiene con la vicina terraferma - rapporto che non muta dopo il progetto di Porto Marghera, manifestando l'aporia della "Grande Venezia" in particolare nel tentativo di risolvere il problema, come vedremo, del sovraffollamento. Al terzo posto c'è la questione edilizia. Nell'inchiesta sanitaria nazionale del 1899 l'ufficio sanitario di Venezia parla di città da risanare (ivi: 187). La città lagunare è ben lontana dal modello igienista; inoltre, a causa delle architetture di pregio, è impossibile procedere con operazioni di sventramento e, a causa della laguna, la città richiede incessanti monitoraggi e operazioni di intervento (Zucconi 2001: 95). Il

${ }^{6}$ Sulla rappresentazione di via Fratelli Bandiera: Cerasi 2007: 63, dove si considera solo il quarto aspetto.

7 | www.ocula.it | agosto 2012 


\section{Ocula ${ }^{13}$}

Architettura e politica: un incrocio di sguardi | Giorgio Coratelli, I luoghi di Marghera Appunti per una geologia urbana

commercio di merci povere in città e la vicina terraferma malsana sono portatori di malattie\%. Infine, sebbene gli aumenti demografici siano modesti, la mancanza di spazi è cronica e il problema del sovraffollamento, specie nelle zone più vicine alle aree portuali e industriali, insieme a quello edilizio - abitazioni fatiscenti, buie, umide e al pianoterra - richiedono interventi radicali8.

Queste tre questioni compongono il "problema di Venezia". La gerarchia con cui sono state esposte descrive l'ordine nel quale si tenta una soluzione correlata. Il "problema di Venezia" appare un problema di governo della città. Si tratta infatti di trovare soluzioni adeguate alle seguenti domande: come rispondere all'incremento di traffico e al problema di deposito delle merci povere? Dove concedere spazi alle industrie, considerando il problema dei collegamenti con il porto e con la ferrovia? La questione edilizia si risolve con la costruzione di nuovi abitazioni; poiché si tratta di abitazioni per il basso ceto, la questione va risolta con quella industriale: dove trovare gli spazi per nuove industrie e abitazioni?

Il periodo più acceso del dibattito va dal 1902 al 1905: in questo periodo si collocano i discorsi dei due principali esponenti dello schieramento a favore della sistemazione del porto in terraferma. Nel 1902, sulla "Gazzetta di Venezia", appare un articolo del capitano Petit in cui per la prima volta si propone di sistemare il porto di Venezia sulla terraferma. Nel 1904 Petit pronuncia un discorso all'Ateneo Veneto in cui riorganizza la sua proposta. Nel 1905 il conte Foscari pubblica un libretto in cui espone gli argomenti a favore della soluzione escogitata da Petit9. Il saggio viene riedito nel 1917, l'anno della fondazione del sito portuale in terraferma. Non è possibile in questa sede ricostruire i termini del dibattito alla luce del problema del governo della città. Considereremo pertanto solo il discorso di Petit e il saggio di Foscari.

In questi due testi le tre questioni che compongono il "problema di Venezia" sono connesse l'una all'altra. Petit (1904: 8-9) afferma che non si può risolvere una questione senza tener conto delle altre: ciò comporterebbe il rischio di soluzioni contrastanti. Il suo discorso si concentra su due punti. Il primo punto è quello della "comunicazione", secondo tre ordini di questioni: vie del mare, lagunari, fluviali, terrestri e ferroviarie; trasporto e transito di merci povere e ricche e di persone; velocità dei trasporti ed efficienza della comunicazione. Il "problema di Venezia", secondo Petit, è in sintesi il rischio dell'immobilismo. La soluzione è nell'incremento efficiente dei flussi dinamici, dei movimenti di cose e di persone. Con "comunicazione" si intende quindi analisi e ricerca delle soluzioni del "problema di Venezia" in termini di velocità ed efficienza di circolazione: circolazione delle merci e delle navi migliorando la logistica portuale e le vie di navigazione (questione

7 Elementi che emergono soprattutto nell'indagine sulla malaria dell'ufficiale sanitario Raffaele Vivante (Vivante 1902).

8 Zucconi (2001: 95) parla di 900 abitanti per ettaro nella zona di Castello rispetto ai 637 di Berlino e ai 620 di Vienna nel XIX secolo.

$9 \mathrm{Nel} 1904$ Foscari tiene una conferenza in cui pone il problema logistico del porto di Venezia in rapporto agli altri porti del mare Adriatico.

8 | www.ocula.it | agosto 2012 


\section{Ocula ${ }^{13}$}

Architettura e politica: un incrocio di sguardi | Giorgio Coratelli, I luoghi di Marghera

Appunti per una geologia urbana

portuale), circolazione delle merci migliorando la comunicazione tra luoghi di produzione e vie di trasporto marittime e terrestri (questione industriale), circolazione delle persone da spostare verso la terraferma con la costruzione di case operaie, dove garantire una migliore circolazione di aria e di luce (questione edilizia e igienica). Il secondo punto è quello dello spazio: per migliorare la circolazione bisogna allargare gli spazi, trovare nuovi spazi se non vi è disponibilità nel centro abitato. Per Petit si tratta di un punto fondamentale soprattutto per la questione edilizia.

Circolazione e spazio sorreggono gli argomenti di Foscari (1917: 28-30), nel cui intervento ciascuna questione è ulteriormente approfondita. Sulla questione portuale, dopo aver dimostrato che l'incremento del traffico delle merci povere ha superato di molto le previsioni, il conte afferma che bisogna sistemare il porto in una zona dove sia possibile un'espansione illimitata della struttura. Riguardo alla questione industriale, afferma la necessità di organizzare una grande zona vicina al porto, dove garantire la concentrazione di capitale e di lavoro (ivi: 68). Affrontando la questione edilizia, Foscari sostiene la costruzione di un sobborgo industriale in terraferma dove spedire la popolazione, la «turba di accattoni» (ivi: 70), sfollata, e risolvere così il problema edilizio e quello morale, in particolare dell'alcoolismo. Le tre questioni sono ben intrecciate: la sistemazione per il porto migliora le comunicazioni con la ferrovia, permette la bonifica delle zone paludose, preserva Venezia e la laguna da ulteriori ristrutturazioni (ivi: 89). È interessante notare come Foscari utilizzi l'argomento ecologico e quello della tutela a favore della propria tesi - laddove ci si interessa alla tutela di Venezia e non del sobborgo industriale; gli stessi argomenti, a partire dagli anni Sessanta, saranno punti centrali della protesta contro le industrie pesanti di Porto Marghera. Inoltre, per la questione edilizia, l'argomento di Foscari si basa su due saperi, demografia e ingegneria sanitaria, effettuando una stima del fabbisogno di case e richiamando le analisi dell'ufficiale sanitario di Venezia, Raffaele Vivante, per quel che riguarda il problema del sovraffollamento. ${ }^{10}$ Sebbene le analisi tecniche di Vivante non si traducano in proposte urbanistiche, l'ingegneria sanitaria funge nel dibattito da sapere discriminante: nei discorsi dei "neo-insularisti", ancorati alla storia e a un'immagine mitica della città, non vi sono argomenti diretti né contro né a sostegno di problematiche igieniche.11

Riassumendo, il "problema di Venezia" concerne il governo della città nel senso in cui impone di risolvere insieme le tre questioni: (1) la questione portuale, con una nuova sistemazione logistica che permetta eventuali futuri sviluppi in relazione all'incremento dei traffici; (2) la questione industriale, per l'istituzione di una "zona franca" vicina al porto; (3) la questione edilizia, con la costruzione di un sobborgo industriale e l'avvio di procedure di

10 Vivante svolge l'inchiesta sulle abitazioni nel 1910. Foscari si riferisce a una precedente inchiesta dell'ufficiale sanitario sulla tubercolosi.

${ }^{11}$ Ciò è particolarmente evidente in Salvadori 1917, il quale afferma la pericolosità della terraferma in quanto zona malarica, senza tenere conto delle necessarie bonifiche per le nuove sistemazioni. In questa sede non è possibile approfondire questo punto e la posizione dei "neo-insularisti". 


\section{Ocula ${ }^{13}$}

Architettura e politica: un incrocio di sguardi | Giorgio Coratelli, I luoghi di Marghera

Appunti per una geologia urbana

sfollamento. Le tre questioni sono attraversate da quattro problematiche trasversali: (a) problematica igienica, relativa allo sbarco di merci povere, all'inquinamento industriale, alla sovrappopolazione e alle endemie; (b) problematica finanziaria, concernente i rendimenti del porto, le concentrazioni industriali e l'occupazione della popolazione povera; (c) problematica strategica, riguardante la logistica, l'efficienza delle vie di comunicazione, la costruzione del sobborgo industriale; (d) problematica politica e simbolica, che comprende il primato del porto di Venezia nell'Adriatico, lo sviluppo industriale e la costruzione di un moderno sobborgo su modello della città-giardino.

I saperi convocati sono ingegneria sanitaria, demografia, economia politica, analisi del commercio. Questi forniscono i dati materiali, strutturati sulla base di quattro aspetti: quantità - precisione dei dati e approssimazione per modifiche relative (per es. il calcolo di fabbisogno di case); qualità - interpretazione dei dati, valutazione di vantaggi e svantaggi riguardo a provvedimenti presi o da prendere; probabilità - i dati sono enunciati in prospettiva del futuro; versatilità - un dato può ritornare in diverse argomentazioni (per es. il quantitativo di merci povere sbarcate rispetto al problema logistico dei depositi e a quello igienico dell'inquinamento atmosferico e lagunare). Nei discorsi di Petit e di Foscari, questi dati sono organizzati intorno alle due direttrici fondamentali, circolazione e spazio, che definiscono lo schema di lettura.

\section{Il governo della città}

Circolazione e spazio rimandano alla nozione di diagramma della sicurezza, o biopolitico, elaborata da Foucault e ripresa da Deleuze. Ma, come vedremo, per la nostra analisi sarà importante anche la nozione di diagramma disciplinare. Passiamo ora a introdurre le due categorie per proseguire, nei due capitoli seguenti, alla descrizione geologica di Marghera e di Porto Marghera.

Il problema dello spazio, scrive Foucault (2004: 21 tr. it.), è centrale per le pratiche di governo. Sovranità, disciplina e sicurezza si distinguono in quanto pratiche che hanno a che fare con una molteplicità nello spazio. Consideriamo solo le pratiche disciplinari e di sicurezza (ivi: 25-28). Il diagramma disciplinare si esercita su una piccola città - spazio ristretto eretta in uno spazio vuoto, secondo un principio di organizzazione gerarchica e di distribuzione funzionale degli elementi. Il diagramma della sicurezza si esercita su uno spazio urbano pre-esistente, da pianificare e ricostruire secondo un principio di distribuzione spaziale che integri le possibilità di sviluppo edilizio, demografico, commerciale, industriale della città. In questo caso, il problema dello spazio urbano è principalmente un problema di circolazione.

Deleuze, nelle sue letture delle analisi di Foucault, definisce il diagramma lo schematismo relativo a ogni formazione storica: il diagramma agisce come causa immanente e coestensiva all'intero campo sociale, è la carta dei 


\section{Ocula ${ }^{13}$}

Architettura e politica: un incrocio di sguardi | Giorgio Coratelli, I luoghi di Marghera

Appunti per una geologia urbana

rapporti di forze propri a una formazione (Deleuze 1986: 54-56, $114 \mathrm{tr}$. it.). Il diagramma disciplinare è l'insieme di funzioni che operano su una molteplicità ridotta e distinta in uno spazio ristretto, mediante l'imposizione di una condotta attraverso la ripartizione nello spazio (funzioni di anatomopolitica) (ivi: 52). La nozione di quadrillage è centrale per l'analisi del processo di disciplinamento: introduce alla descrizione dell'organizzazione funzionale e gerarchica dello spazio chiuso, che si estende fino al dettaglio, e dell'utilizzazione esaustiva dei singoli corpi (ivi: 53; v. Foucault 1975: 213218 tr. it. sul modello della città appestata). Il diagramma di sicurezza è l'insieme delle funzioni che operano su una molteplicità qualunque e numerosa, una popolazione, in uno spazio aperto ed esteso, mediante la riproduzione di condotte molteplici e probabili attraverso la serializzazione nello spazio (funzioni di bio-politica) (Deleuze 1986: 100; Foucault 1976: 123-124 tr. it.). La nozione di serie è centrale per l'analisi del processo biopolitico: introduce alla descrizione della gestione e della regolazione dei flussi nello spazio aperto, che si estendono in funzione di possibilità che occorre calcolare, e della loro distribuzione efficiente - da cui l'importanza della logistica e delle vie di comunicazione (Foucault 2004: 29).

Un diagramma, scrive Deleuze (1986: 56-57), si attualizza, integra e differenzia nei suoi effetti: il diagramma disciplinare in un ambito delimitato, in uno spazio ristretto; il diagramma biopolitico in uno spazio aperto ed esteso che garantisce il possibile sviluppo delle attività.

\section{Per una geologia di Marghera}

Nel progetto di Emilio Emmer (1922), la forma del sobborgo di Porto Marghera è una "libera" traduzione della città-giardino di Ebenezer Howard, solo della sua forma urbana ${ }^{12}$. Il quartiere di Marghera, in quanto sobborgo industriale, rimanda al modello della città operaia, presente anche nel Veneto (v. Guiotto 1979; Maifreda 2007: 88-91). Seguendo queste due coordinate, si può dire che nel progetto di Marghera, come nella città operaia, si intersecano meccanismi disciplinari agenti sul corpo e meccanismi regolatori in funzione della popolazione (v. Foucault 1997: 216217 tr. it.). Il meccanismo disciplinare agisce sulle abitazioni, definite secondo un'organizzazione gerarchica e funzionale: case isolate o edifici bassi per operai, case isolate per impiegati, villini per i dirigenti (Emmer

12 Howard 1902. L’idea di città-giardino è stata modello per molti progetti urbanistici. Ma il testo di Howard è interessante anche per un altro aspetto: a metà fra "sogno disciplinare" e "sogno biopolitico", inventa la progettazione della città preoccupandosi soprattutto della sua economia politica. Contiene in un solo capitolo le proposte urbanistiche, per il resto affronta questioni riguardanti l'amministrazione della città, il valore dei terreni, contabilizzando vantaggi e svantaggi dell'abitare. Non si tratta propriamente di utopia: Howard intende mostrare come la costruzione di una città-giardino dipenda da buone pratiche di controllo e di regolazione, dal monitoraggio assiduo delle strutture e dai rendimenti dei profitti re-investiti. Eseguendo con cura queste operazioni si può, secondo Howard, ristrutturare completamente Londra.

11 | www.ocula.it | agosto 2012 


\section{Ocula ${ }^{13}$}

Architettura e politica: un incrocio di sguardi | Giorgio Coratelli, I luoghi di Marghera

Appunti per una geologia urbana

1922); giardini o orti privati per ogni casa, utili alla normalizzazione e moralizzazione delle famiglie (Guiotto 1979: 71). Il meccanismo regolatore riguarda lo spazio urbano, il reticolo che unisce le case: si tratta innanzitutto di prendere misure igieniche per fornire condizioni materiali migliori di Venezia - giardini ed edifici pubblici, rifornimenti di acqua potabile. Nel progetto di Emmer, inoltre, il reticolo stradale non segue il tracciato perpendicolare, preferito nelle città operaie, ma il sistema delle rotonde, proprio della città-giardino.

Diagramma disciplinare e diagramma biopolitico articolano il quartiere urbano: bisogna risolvere la questione edilizia (tratto biopolitico), ma ciò può essere fatto solo costruendo un quartiere nuovo su uno spazio vuoto (tratto disciplinare); il sovraffollamento di Venezia può essere risolto concentrando la popolazione in un nuovo quartiere (tratto biopolitico) seguendo procedure di organizzazione gerarchica e funzionale (tratto disciplinare).

Dopo pochi anni il progetto sembra già fallito: mancano i finanziamenti e non si avviano procedure di sfollamento da Venezia (Facca 2004: 53). Da un punto di vista metodologico, ciò permette di osservare l'attualizzazione del diagramma nei suoi effetti non come l'applicazione di un piano o l'elaborazione di procedure di governo, ma secondo una dinamica complessa, di intensità delle forze in gioco, di tensioni tra forze e resistenze.

Schematizzando, nel caso di Marghera possiamo in linea generale osservare tre aspetti di queste dinamiche. Le forze si scontrano con altre forze: gli industriali, contrariamente a quanto pattuito all'atto di fondazione di Porto Marghera, non ingaggiano lavoratori o disoccupati veneziani, ma manodopera contadina perché più resistente e meno turbolenta. Le forze di governo perdono intensità: le procedure biopolitiche non riescono a governare né a condizionare il mercato del lavoro: a Porto Marghera si diffonde l'uso degli stagionali, il che va a vantaggio dei contadini; quando il prefetto richiama ai patti gli industriali, le forze di questi si rivelano maggiori di quella dell'autorità pubblica ${ }^{13}$. Vi sono poi altre resistenze: quelle dei veneziani, restii a trasferirsi sulla terraferma (Piva 1991: 36).

Fallisce il proposito di concentrare la popolazione lavorativa proveniente da Venezia in un sobborgo industriale per avvicinare abitazione e nuovo luogo di lavoro' ${ }^{14}$. Marghera non diventa né un sobborgo-giardino né un sobborgo industriale. Si cerca di ovviare a ciò con delle forzature:

13 Nel 1927 i disoccupati di Mestre protestano contro l'ingaggio dei contadini chiedendo lavoro nelle fabbriche. Negli anni Trenta le proteste si allargano ai disoccupati veneziani (si noti che i veneziani non disdegnano il lavoro nelle fabbriche di Porto Marghera: rifiutano di lasciare le abitazioni lagunari, chiedono un servizio di trasporto per la terraferma - il Ponte Littorio, poi Ponte della Libertà, che collega Venezia con il suo entroterra, viene inaugurato nel 1933). Nel 1932 interviene il prefetto di Venezia, il quale decreta che nell'assunzione vanno privilegiati i cittadini rispetto ai contadini. Ma gli industriali si oppongono a tale provvedimento, anche con metodi illeciti di iscrizione dei contadini agli uffici di collocamento (Piva 1991: 37).

14 Così Emilio Emmer: «la formazione del nuovo sobborgo in terraferma verrà indubbiamente a determinare un esodo di lavoratori dalla città al sobborgo, per la convenienza di avvicinarsi al luogo di lavoro» (Emmer 1922: 9). 


\section{Ocula $\mathbf{a}^{13}$}

Architettura e politica: un incrocio di sguardi | Giorgio Coratelli, I luoghi di Marghera Appunti per una geologia urbana

l'edificazione tra il 1929 e il 1938 di tre villaggi, Ca' Emiliani il primo, poi Ca' Sabbioni e Ca' Brentelle per gestire lo sfollamento da Venezia e l'urgente problema delle case in seguito alla liberalizzazione degli affitti, decretata nel 1926 (Facca 2004: 64-66). I tre villaggi sorgono distanti dal centro abitato di Marghera, concepiti per la popolazione deportata da Venezia. Sono composti di alloggi molto piccoli - due stanze, di cui una cucina con lavabo, mentre la latrina è all'esterno - e senza pavimento, costruiti con materiale scadente, non dotati di servizi. In ogni villaggio vi è un centro di sorveglianza diretto da un custode, coadiuvato da carabinieri.

Questi villaggi rimandano a meccanismi disciplinari, ma sembrano riprodurre anche meccanismi di sovranità (Foucault 2004: 23-24): l'esercizio del potere proviene dal centro e deve radicarsi in ogni angolo dello spazio ristretto. Il custode esercita anche il potere economico di distribuzione: riceve dal Comune e fornisce agli abitanti i semi necessari per trasformare i terreni incolti, sui quali sorgono le case, in orti e giardini. Questi villaggi possiedono un loro centro, emanazione diretta del potere centrale, non hanno contatti con la popolazione del quartiere urbano. Si parla di villaggi-ghetto in quanto la popolazione vi vive come in confino, sottoposta a un "potere dominante" che si occupa esclusivamente di questi luoghi15: le procedure forzate di esilio di veneziani poveri (e di alcune famiglie di antifascisti obbligati a lasciare Venezia) hanno lo scopo di incentivare l'emigrazione di classi popolari dal centro verso le periferie e incoraggiare il trasferimento di classi borghesi verso il centro dalle periferie, in modo da differenziare e uniformare il valore delle case ${ }^{16}$. Inoltre, i villaggi diventano fin da subito, e lo sono tuttora, oggetto di valorizzazione negativa: lontani dal centro, sono visti come luoghi pericolosi, popolati da disoccupati e delinquenti ${ }^{17}$.

Il fallimento del progetto del quartiere-giardino comporta non solo l'installazione di villaggi-ghetto lontani dal centro abitato, ma anche la costruzione di case popolari senza più rispettare il piano iniziale, processo che prosegue negli anni del secondo dopoguerra (Cogo 2003). Si delinea così il frastagliato paesaggio di Marghera, composto di villette, baracche, palazzine costruite con materiali poveri, edifici di più di tre piani, lungo un reticolo stradale che solo in parte segue il tracciato delle rotonde (Nappi 1994: 80).

La nascita e gli sviluppi di Marghera mostrano come l'attualizzazione del diagramma nei suoi effetti non sia un semplice dato applicativo, ma dipende: dai rapporti di forze in gioco - per es. industriali e politici, ma anche industriali e lavoratori; dalle intensità delle forze - nel caso di Marghera si è accennato alla debolezza delle forze pubbliche nel gestire, controllare, contrattare con le forze private; dal rapporto tra forze e resistenze - gli

$15 \mathrm{Vi}$ è qui una differenza rispetto a quanto scrive Crivelli sulle borgate romane (2008: 73-74) come espressioni del solo modello disciplinare ed estranee al potere dominante.

${ }^{16}$ Come affermato dall'ingegnere Miozzi, capo dell'ufficio tecnico del comune (cit. in

Facca 2004: 64).

${ }_{17} \mathrm{Si}$ vedano le interviste raccolte in Cerasi 2007. 


\section{Ocula ${ }^{13}$}

Architettura e politica: un incrocio di sguardi | Giorgio Coratelli, I luoghi di Marghera

Appunti per una geologia urbana

industriali preferiscono i contadini ai veneziani perché più adatti ai lavori pesanti e più pacifici. Secondo questa dinamica, l'integrazione del diagramma nei suoi effetti avviene per deviazioni: la preferenza di manodopera contadina da parte degli industriali è tra le cause del fallimento del progetto del quartiere giardino, e questo conduce alla decisione di procedere allo sfollamento da Venezia e alla costruzione dei villaggi-ghetto. La molteplicità diagrammatica si attualizza seguendo diverse linee di differenziazione. Nella prima fase di industrializzazione ciò comporta la coesistenza di due dualismi coestensivi: quello del sobborgo giardino (traduzione dei modelli della città-giardino e della città operaia in un progetto al quale corrisponde un primo tracciato del quartiere) e quello dei villaggi-ghetto (traduzione del modello delle borgate romane in progetti ai quali corrispondono i tre villaggi).

\section{Per una geologia di Porto Marghera}

Passiamo ora dalla zona residenziale alla zona industriale. Si è visto come il problema del porto di Venezia fosse soprattutto un problema di logistica. Nei discorsi dei sostenitori della sistemazione in terraferma si parla molto della necessità di sbloccare i limiti allo sviluppo. Coen Cagli (1923: 256), progettista del nuovo porto, espone una dettagliata analisi tecnica delle soluzioni logistiche ottenute con il porto in terraferma. Il problema, tuttavia, non riguarda solo il porto, ma anche le industrie: Porto Marghera nasce per diventare un polo di sviluppo trainante dell'economia veneta e nazionale.

A tal proposito, si possono riprendere gli studi di Hirschman (1958 e 1977) sulle economie di sviluppo. L'economista tedesco oppone a un'economia pianificata un più vantaggioso sviluppo che procede "in sequenze" e per vie trasversali, a partire da una qualche attività produttiva trainante. A tale proposito Hirschman (1977: 100 tr. it.) elabora il concetto di connessione. In rapporto a un'attività produttiva trainante, si parla di connessioni di produzione quando si incentiva lo sviluppo di attività che forniscono gli input (connessioni a monte) e di attività che ne utilizzano gli output (connessioni a valle). Nel caso di Porto Marghera, questi processi si possono individuare nel completamento dei cicli di produzione da parte di alcuni stabilimenti ${ }^{18}$. Si parla invece di connessioni di consumo quando l'effetto si riproduce su attività produttive non collegate a quella trainante. Questi effetti possono essere positivi, negativi o inesistenti. Nel caso di Porto Marghera, un esempio di connessione di consumo positiva è il rapporto indiretto tra industria elettrica e industria chimica; un esempio di connessione di consumo negativa è il rapporto tra industrie pesanti e agricoltura; un esempio di connessione di consumo inesistente è il rapporto tra industria elettrica e industria alimentare. Un terzo ordine di connessioni è quello della connessione fiscale, che comprende imposte e flussi di reddito.

${ }^{18}$ Negli anni Cinquanta la Sava completa il ciclo dall'alluminio alle lamiere e la SiceEdison completa il ciclo del cloro (Favaro 2008: 18-21). 


\section{Ocula $\mathbf{a}^{13}$}

Architettura e politica: un incrocio di sguardi | Giorgio Coratelli, I luoghi di Marghera Appunti per una geologia urbana

Queste connessioni non corrono parallele ma si intersecano fra loro. Per esempio, la connessione fiscale produce effetti positivi sulle connessioni di consumo nella fase di apertura degli stabilimenti a Porto Marghera: negli anni Trenta, grazie al ruolo strategico del conte Volpi (ministro delle Finanze), e negli anni Sessanta, nel periodo di apertura della seconda zona industriale, a favore delle società dell'Edison e della Montecatini (v. Dorigo 1973: 169, 176-178; Chinello 1979: 192-200 e Chinello 1975: 205-206). È importante dunque tener conto delle posizioni strategiche degli attori in gioco.

Hirschman (1977: 122-130) arricchisce questo quadro economico con altre due connessioni: in primo luogo le connessioni tecnologiche che riguardano le tecniche di produzione e di lavoro; un esempio di tali connessioni a Porto Marghera negli anni Cinquanta è l'introduzione da parte della Sice-Edison delle tecnologie per la produzione di PVC, che segna l'avvio delle grandi produzioni della chimica (Favaro 2008: 21). In secondo luogo le connessioni politiche. Queste possono in parte derivare dagli incroci tra connessioni fiscali e connessioni economiche, alla luce - come si è detto sopra - di posizioni e strategie di attori economici e attori politici. Ma queste connessioni possono anche manifestarsi in altri rapporti. Hirschman (1984: 230-233 tr. it) collega le dinamiche descritte dalle connessioni e le funzioni di voce, uscita, lealtà (Hirschman 1970). Lo fa in particolare alla luce del concetto di "margine per le prestazioni scadenti": ogni fase del processo economico (a livello generale come nel dettaglio dell'operazione da compiersi nella prestazione lavorativa) ha un limite di efficienza e qualità di esecuzione. Superato questo limite, come scrivono Deleuze e Guattari (1980: 610-611) c'è una soglia che fa cambiare connessione: si può allora passare dalla connessione economica alla connessione politica. È ciò che accade nel periodo di crisi della fase industriale con le lotte, soprattutto nel Petrolchimico, contro le dure condizioni di lavoro. In tale ambito si pone il problema dell'intersecarsi del diagramma disciplinare, che riguarda lo spazio ristretto della fabbrica, con il diagramma biopolitico, che concerne lo spazio esteso della zona industriale. Ciò permette di mettere in relazione il progetto per una terza zona industriale e le lotte che hanno fatto decadere tale progetto, le resistenze operaie che agiscono nello spazio disciplinare e quelle ambientaliste che agiscono nello spazio biopolitico ${ }^{19}$.

È proprio dentro questa fase di lotta che emerge il problema della nocività della fabbrica sotto il suo aspetto politico ${ }^{20}$. Nella lunga durata di

19 Promotore del progetto per una terza zona industriale è Wladimiro Dorigo (1973: 180-201 dove controbatte alle critiche mosse da parte dell'associazione Italia Nostra). Gli ambientalisti si battono per la salvaguardia di Venezia, in particolare dopo l'alta marea del 1966. A questa battaglia partecipa anche Indro Montanelli (gli articoli scritti "per Venezia" sul "Corriere della Sera" - 22, 23, 24 e 26 novembre 1968 - sono disponibili all'indirizzo www.territorioveneto.it/ reprints21.htm).

${ }^{20} \mathrm{Si}$ vedano le pagine di Sbrogiò sugli anni Settanta a Porto Marghera, in particolare sulle riviste Lavoro zero e Controlavoro (Sacchetto e Sbrogiò 2009: 71-76, 99-103), i dati sulle fughe di gas nel Petrolchimico tra il 1971 e il 1973 raccolti da Chinello (Chinello 1975: 249-274) e da Gabriele Bortolozzo, l'operaio che, denunciando i dirigenti della Montedison, ha dato avvio al procedimento giudiziario negli anni 


\section{Ocula $\mathbf{a}^{13}$}

Architettura e politica: un incrocio di sguardi | Giorgio Coratelli, I luoghi di Marghera

Appunti per una geologia urbana

questa lotta, che allo stesso tempo avvicina e allontana Porto Marghera e Marghera, si possono riscontrare momenti di protesta (in particolare la manifestazione del 1974 contro le lavorazioni nocive al Petrolchimico, con il manichino operaio issato sulla croce $^{21}$ ), di esodo dalla fabbrica, di manifestazioni di lealtà - sindacati e operai che accettano la monetizzazione del rischio temendo la chiusura degli stabilimenti. Un'altra soglia importante che caratterizza i processi economici di Porto Marghera è quella che si può definire "soglia ambientale" e che rimanda ai conflitti più recenti - fase postindustriale - sulla tutela dell'ambiente e della salute (v. Fabbri 2003; Benatelli, Candiello, Favarato 2006).

Il modo in cui le connessioni politiche agitano il quadro economico sottolinea, ancora una volta, l'importanza dei modi di manifestazione dei rapporti di forze. Si possono quindi mettere in relazione le declinazioni del concetto hirschmaniano di connessione con il concetto di serie di Foucault e con il rapporto tra limite e soglia sviluppato da Deleuze e Guattari. Ciò permetterebbe di tracciare una mappa dinamica degli eventi storici di Porto Marghera, e di mostrare come le configurazioni diagrammatiche - problema ecologico sul piano biopolitico e problema delle condizioni di lavoro sul piano disciplinare - si intreccino e attualizzino in forme molteplici anche nella zona industriale di una città.

\section{Bibliografia}

Baiocco, R.

2002 "Progetti per il nuovo porto dei Bottenighi”, in: G. Zucconi (a cura di), $L a$ Grande Venezia. Una metropoli incompiuta tra Otto e Novecento, Venezia, Marsilio, 2002, pp. 41-49.

Barizza, S.

2004a "Da Bottenigo (frazione di Mestre) a Marghera (frazione di Venezia)", in: Barizza 2004b.

2004b (a cura di), Marghera. Il quartiere urbano, Marghera, Alcione Editore.

2009 (a cura di), Marghera 2009. Dopo l'industrializzazione, Marghera, Alcione Editore.

Benatelli, N., Candiello, A., Favarato, G.

2002 Processo a Marghera: l'inchiesta sul Petrolchimico il CVM e le morti degli operai. Storia di una tragedia umana e ambientale, Portogruaro, Nuova Dimensione.

2006 Laboratorio Marghera tra Venezia e il Nord est: la giurisprudenza ambientale, la partecipazione attiva dei cittadini, le bonifiche e le prospettive di sviluppo, Portogruaro, Nuova Dimensione.

Novanta (Bortolozzo 1998: 145-147, 153-156, 267-273). Già nel 1974 la casa editrice Bertani di Verona pubblica un libretto che denuncia i rischi per i lavoratori delle fabbriche e per la popolazione civile (Moriani 1974). La nocività è ora considerata sotto il suo aspetto politico, ma era già evidente sotto l'aspetto simbolico: si veda per es. quel che Bortolozzo scrive sulla Sice-Edison, battezzata dagli operai "il sanatorio" (1998: 32-33).

${ }^{21}$ L'evento venne organizzato con l'artista Giovanni Rubino e il poeta Corrado Costa (sul cui sito www.farememoria.it sono pubblicate le foto dell'happening). 


\section{Ocula $\mathbf{a}^{13}$}

Architettura e politica: un incrocio di sguardi | Giorgio Coratelli, I luoghi di Marghera

Appunti per una geologia urbana

Bortolozzo, G.

1998 L'erba ha voglia di vita. Autobiografia e storia politica tra laguna e Petrolchimico, Venezia, Associazione G. Bortolozzo.

Casson, F.

2007 La fabbrica dei veleni. Storie e segreti di Porto Marghera, Milano, Sperling \& Kupfer.

Cerasi, L.

2007 Perdonare Marghera, Milano, Franco Angeli.

Chinello, C.

1975 Storia di uno sviluppo capitalistico. Porto Marghera e Venezia 1951-1973, Roma, Editori Riuniti.

1979 Porto Marghera 1902-1926. Alle origini del problema di Venezia, Venezia, Marsilio.

Coen Cagli, E.

1923 "Il porto di Venezia", in: Rivista mensile di Venezia, 11, 1923.

Cogo, C.

2003 "Le vicende urbanistiche di Marghera tra il 1938 e il 1955", in: S. Barizza (a cura di), Marghera 1938-1955, Marghera, Alcione Editore, 2003.

Cervelli, $\mathrm{P}$.

2008 "Effetto-margine: spazi urbani e periferie culturali", in G. Marrone, I. Pezzini (a cura di), Linguaggi della città. Senso e metropoli II. Modelli e proposte di analisi, Roma, Meltemi, 2008.

Deleuze, G.

1986 Foucault, Paris, Minuit (tr. it., Foucault, Napoli, Cronopio, 2002).

Deleuze, G., Guattari, F.

1980 Mille plateaux. Capitalisme et schizophrénie, Paris, Minuit (tr. it., Mille piani. Capitalismo e schizophrenia, Roma, Castelvecchi, 2003).

Dorigo, W.

1973 Una legge contro Venezia. Natura, storia, interessi nella questione della città e della laguna, Roma, Officina.

Emmer, P., E.

1922 "Il quartiere urbano di Porto Marghera (il nuovo sobborgo giardino di Venezia in terraferma)", in: Rivista mensile di Venezia, 5, 1922.

Fabbri, F.

2003 Porto Marghera e la laguna di Venezia: vita, morte, miracoli, Milano, Jaca Book.

Facca, G.

2004 "Marghera, nascita di un quartiere", in Barizza 2004 b.

Favaro, O.

2008 Un cardellino in gabbia. Fabbrica e lavoro nei primi anni Cinquanta a Porto Marghera, Mestre, StoriAmestre.

Foscari, P.

1917 Per il più largo dominio di Venezia. La città e il porto, Milano, Fratelli Treves Editori.

17 | www.ocula.it | agosto 2012 
Foucault, M.

1975 Surveiller et punir. Naissance de la prison, Paris, Gallimard (tr. it., Sorvegliare e punire. Nascita della prigione, Torino, Einaudi, 2006).

1976 La volonté de savoir, Paris, Gallimard (tr. it., La volontà di sapere. Storia della sessualità 1, Milano, Feltrinelli, 2008).

1997 Il faut defendre la sociéte, Paris, Gallimard (tr. it., Bisogna difendere la società, Milano, Feltrinelli, 2009).

2004 Sécurité, territoire, population. Cours au Collège de France (1977-1978) (tr. it., Sicurezza, territorio, popolazione, Milano, Feltrinelli, 2005).

Giovannini, C.

1996 Risanare la città. L'utopia igienista di fine Ottocento, Milano, Franco Angeli.

Guiotto, L.

1979 La fabbrica totale. Paternalismo industriale e città sociali in Italia, Milano, Feltrinelli.

Hirschman, A., O.

1958 The strategy of economic development, New Haven, Yale University Press (tr. it., La strategia dello sviluppo economico, Firenze, La Nuova Italia, 1968).

1970 Exit, voice and loyalty: responses to decline in firms, organizations and states, Cambridge, Harvard University Press, (tr. it., Lealtà, defezione, protesta, Milano, Bompiani, 1982).

1977 "Un approccio allo sviluppo basato sulla generalizzazione dell’idea di connessione, con particolare riferimento agli staples”, Economic development and cultural change, 25, pp. 67-98 (tr. it., Hirschman 1992, pp. 95-135).

1984 "La confessione di un dissenziente. La strategia dello sviluppo economico rivisitata”, in G. Meier e D. Seers (a cura di), Pioneers of economic development, New York, Oxford University Press, 1984 (tr. it. Hirschman 1992).

1992 Ascesa e declino dell'economia dello sviluppo, a cura di A. Ginzburg, Torino, Rosenberg \& Sellier.

Howard, E.

1902 Garden cities of To-morrow, Faber and Faber, London (tr. it., La città giardino del futuro, Bologna, Calderini, 1972).

Maifreda, G.

2007 La disciplina del lavoro: operai, macchine e fabbriche nella storia italiana, Milano, Bruno Mondadori.

Moriani, G.

1974 Nocività in fabbrica e nel territorio, Verona, Bertani.

Nappi, A., F.

1994 Storia di Marghera. Da periferia a città, Marghera, Centro sportivo culturale Catene.

Petit, L.

1904 "Sistemazione del porto di Venezia in relaziona a più facili ed economiche comunicazioni colla terraferma”, Memoria letta all'Ateneo Veneto nell'adunanza accademica del 21 gennaio 1904, Venezia, Tipografia orfanotrofio di Antonio Pellizzato.

Piva, F.

1991 Contadini in fabbrica. Il caso Marghera 1920-1945, Roma, Edizioni Lavoro.

Sacchetto, D., Sbrogiò, G. (a cura di)

2009 Quando il potere è operaio, Roma, Manifesto Libri.

18 | www.ocula.it | agosto 2012 


\section{Ocula ${ }^{13}$}

Architettura e politica: un incrocio di sguardi | Giorgio Coratelli, I luoghi di Marghera Appunti per una geologia urbana

Salvadori, A.

1917 Per una "più grande Venezia" protesa verso il mare e contro il "porto di Mestre", Venezia, Premiate Officine Grafiche Carlo Ferrari.

Sbrogiò, I.

1990 Tuberi e pan secco, Venezia, Poligrafo.

Vivante, R.

1902 La malaria in Venezia, Torino, Stabilimento Fratelli Pozzo.

Volpi, G.

1939 Venezia antica e moderna, Roma, Atena.

Zucconi, G.

2001 "Venezia di fronte agli imperativi dell'igienismo", in: F. Cosmai, S. Sorteni (a cura di), L’ingegneria civile a Venezia, Venezia, Marsilio. 